\title{
Current Situation of Higher Education after the Implementation of the National Standard of Students' Physical Health
}

\author{
Lanping Hang, Chunna Zhao, Rui Zhang, Liyun Han \\ Department of Physical Education Northwest Polytechnic University, Xi'an, Shaanxi, 710072, China \\ Email: hlp008@126.com
}

\begin{abstract}
Keywords: National Standard of Students' Physical Health; physical tests; problems and coping strategies
\end{abstract}

\begin{abstract}
There has been numerous researches on the implement ation of the National Standard of Students' Physical Health since 2007. This paper intends to analyze the current situation by means of literature review, and with reference to the writer's working experience and knowledge of physical tests, and proposes corresponding coping strategies.
\end{abstract}

\section{INTRODUCTION}

The recent 20 years have witnessed a continuing decrease in a number of teenage students' physical constitution attributes according to research with an obvious superiority of the key university students over the ordinary ones [1], a speedy increase in super obesity rate with 1/4 male students in cities, and in poor vision rate with $2 / 3$ secondary school students and $83 \%$ college students [2]. In 2007, the average score of students' physical test in Tsinghai University is less than 70 points; the excellent rate is $32 \%$, while $25 \%$ of the student testers fail. In the meantime, the test results demonstrate a common degree of students' height and weight, vital capacity, cardiovascular endurance, and upper limb power, and a low degree of their speed and lower limb power. In general, the physical constitution of the female students is comparatively better than the male students [3]. The 2010 test data positively foresee an upward trend in students' vital capacity nationwide and elementary and secondary students' physical constitution after a 20-year decline. However, their overall physical condition has not been improved evidently. Although the government has been upgrading and perfecting the Standard of Students' Physical Health, the real physical situation of students is unpromising [4]. A major reason explored by some research is that the scoring standard is raised too high, which results in male students' high failure rate and low excellent rate and female students' low excellent and failure rates [5].

The government authority has been constantly adjusting the standard of students' physical health test ever since the Standard was on trial in 2002, but the effect has remained modest. Evidently, there has been no obvious better change in students' physical quality since the implementation of the Standard.

\section{THE STANDARD'S INFLUENCE ON THE TEACHING MODE OF HIGHER PHYSICAL EDUCATION}

Since the implementation of the Standard, students have been provided opportunities to select sports online in accordance to their preference for their PE course in a majority of universities. A $\mathrm{PE}$ course in some universities is composed of one or two compulsory sports and a selective sport; sports theory becomes part of the teaching content with the purpose of equipping students with substantial relative knowledge and laying a good foundation for life-long sport. Some universities take into account students' physical test scores while marking them in the finals of their PE course, encouraging them to exercise and keep fit. Teachers consequently are developing themselves in professional knowledge and teaching capacity.

The Standard has aroused teachers' greater concern about the introduction of exercising methods in addition to the instruction of sporting skills on PE class. In accordance with The Standard, 
university PE teachers in their classes set as their educational purposes constitution enhancement, physical and mental health boost, cultivation of the sense and habit of life-long sport, and development of the capability of self-evaluating health state and sports culture appreciation, by means of scientific and feasible physical exercises and education. The appearance of the sporting skill test as a result of The Standard has changed the university PE course into the one that directs and encourages students to self-consciously participate in doing sports, which contributes to an all-round development of students' constitution. From the perspective of management, some experts have proposed strategies of human management, capital allocation, fields, equipments, time and resource utility, which can help fulfill the above-mentioned aims [6].

\section{PROBLEMS FOUND DURING THE STANDARD'S IMPLEMENTATION}

\section{A. Test Item Problems}

Researchers identified an unclear test item classification in the Standard and a little change in assessment levels; the assessment standard yet is leveled up drastically, which causes an evident increase of test failure percentage [7].

Physical shape and function There is a big rating class difference in height and weight, which is not scientific and reasonable [8]. It appears simple to take height and weight as the physical shape test metrics for they can not be considered the only judging factors of overweight. A negative correlation can be seen between most of the test items in the Standard and weight so that the overweight students account for a larger proportion of the number of students who fail the test. Lung capacity test is used for examining static pulmonary ventilation function. However, it is favorable to make a dynamic test due to the fact that some sick people may show a normal lung capacity level. Additionally, lung capacity test only cannot demonstrate one's lung function level. Thus, the test at present fails to accurately measure students' physical status.

Stamina sports Step test, a popularly-taken test means of students' physical condition, is time-consuming and less feasible. Besides, that individual difference is not taken into account results in unreliable statistics [9]. While a number of students are taking the test at the same time, there may be some who are not able to coordinate with the rhythm, and there may be errors of different degrees in heart rate test results after exercise load. Accordingly, the results fail to appear justified [10]. Experts believe that step test which focuses on cardiovascular endurance and endurance running test which focuses on aerobic endurance are different in nature, and the test scores show an artificial symmetric relationship that is not innate. As a result, the two tests can not be interchangeable when it comes to evaluating cardiovascular efficiency [11]. In practice, most of the students appear fearful of endurance running so that their performance cannot truly reflect their physical state. By contrast, students tend to feel relaxed when doing step test, and can usually perform well, which is one of the reasons why a larger number of students can pass the test.

Flexibility and strength sports Experts point out that in the Standard, some sports that are not much interrelated, like sit and reach, sit-up, gripping, medicine ball throwing and pull-up, are still grouped together as alternatives [7]. The students' physical health test in Ning Bo University in 2007 indicates that students' physical flexibility shown by sit and reach test makes little sense because the fact that the female has superiority over the male in physical flexibility is not fully considered [8]. In this case, the problem that the poor-interrelated sports are grouped together leads to a significant difference of test results.

Speed and dexterity sports Rope skipping, basketball, football, and volleyball are all test sports that reflect testees' comprehensive physical quality and athletic ability. It is not scientific to mix them up with $50 \mathrm{~m}$ running and standing long jump that demonstrate testees' anaerobic capacity and low limb strength. for they cannot well reflect testees' speed and degree of flexibility.

\section{B. Non-test-item Problems}

Objective problems First, the test instruments show a high error rate, and the instruments of the same kind also show a certain error rate between each other. Second, the software for data submitting creates a series of problems like that students' information is entered repeatedly, that 
students' date of birth is unrelated to their ID numbers, that the import of students' information and the export of the test results are time-consuming, that the automatic scoring function and the test item selecting function are not perfected, and that the statistic module and data backup module are lacking [12]. In 2010, the statistics submitted by 6871 universities show the 8 major problems as follows: unreal contact information, inconsistency of the information the university submits with that shown on the website, insufficient effective data, incomplete contact information, incorrect computer time, empty files, non-national database software, and incorrect registration data format [13]. The test instruments universities own are generally long and frequently used, which leads to wastage at different degrees. A high percentage of make-up tests arise from the instruments' malfunction due to the inaccessible after-sale service. What is more, the instruments are used less frequently after being fixed. Other problems include the difficult operation of the software that is used for data submitting, high workload of data submitting, and the lack of statistic modules.

Subjective problems First, the lack of attention. Students are unclear about the Standard's objectives; hence do not take the test seriously and carefully, so that the test results appear inaccurate. Second, the test management is difficult. Since some teachers do not supervise on the test actively, irregularities such as opportunism and cheating happen occasionally. Third, a test on special students is neglected, which makes them unable to know their health state. Whether the physical test can be implemented effectively largely depends upon whether it is attached great importance to by the authority. Some universities go through the motions of the test because of the poor supervision of the higher authority.

\section{SUGGESTIONS AND COPING STRATIGIES}

\section{A. About the Test Items}

The biggest problem is the low degree of correlation among the test items of the same kind. It is suggested that the test items be focused. As for the height and weight items, age difference has to be taken into consideration. It is preferable to refine the division of range, and lower the scores of different range periods. There is supposed to be particular test items for the unhealthy and disabled students so as to provide them with the knowledge of their health state.

\section{B. It is urgent to solve the problem of test instruments.}

It is to ensure the instruments of high quality be produced by qualified enterprises. The after-sale service has to be improved as well. The professional testers in the university are called for, who have a good knowledge of test instruments and can expertly operate them, and are capable of the data submitting work.

\section{It is important to popularize the Standard among university students.}

If students are able to penetratingly understand the test objective and significance, they will change their indifferent attitude to it. It is therefore advocated that the campus network be made best use, so that students can have easy access to their test results and know their physical condition. In the meantime, teachers are suggested to propose sports prescription according to students' test statistics.

\section{Reward and punishment system should be enforced.}

Students gaining distinction have to be rewarded while their PE teachers and tutors deserve rewards as well. As for students with failure, not only are they required to take the make-up test, but to be punished in a proper way. For instance, the test results can be an influential factor of students' final scores for their PE course. Besides, the university authority has to attach greater importance to the Standard's implementation for it positively influences the launching of the physical test. 


\section{References}

[1] L. Y. Chen. "Research on National Students Physical Health Standard.” Sports Culture Guide. 2011.

[2] X. Y. Lai, H. J. Liu, S. L. Liu. “Causes and restraining measures for the constant deterioration of the constitution of teenagers in China.” Journal of Physical Education. No. 5, pp. 125-128, 2007.

[3] Y. Z. Ma. "Testing result and analysis of National Students Physical Health Standard of Tsinghua University in 2007.” China Sport Science and Technology. No. 5, pp. 130-134, 2009.

[4] L. Y. Chen. "Research on National Students Physical Health Standard.” Sports Culture Guide. 2011.

[5] J. M. Liu, Y. Xing, H. Z. Guo. "Research on the rationality of college students' evaluation system base on the National Students’ Physical Health Standard.” Sports Culture Guide. No. 3, pp. 102-104, 2012.

[6] Y. J. Lu. "Reflection on the implementation of the National Students' Physical Health Standard test in universities from the angle of management.” College Education. No. 4, pp.15-17, 2012.

[7] Y. Y. Zhen, A. L. Fang, J. M. Cai. "Comparative study of National Students Physical Health Standard and Students Physical Health Standard (on trial).” Sport Science. No. 7, pp. 92-96, 2009.

[8] J. Y. Li, Q. L. Wu. "Experimetn on the implementation of evaluation indexes in the National Students’ Health Standard.” Journal of Physical Education. No. 9, pp. 68-73, 2009.

[9] D. Y. Liu, D. K. Cao, R. N. Li. "Research and suggestion on step experiment in the National Students’ Health Standard.” Sport Science. No. 10, pp. 54-55, 2004.

[10] R. J. Cai. “A comparative study on the Chinese Students' Physical Health Standard and the American Physical Best Standard.” Journal of Chendu Institute of Physical Education and Sports. No. 4, pp.91-94, 2011.

[11] Z. X. Zhou, G. Ji, F. Zhang. "Experimental study on homogeneity and reliability of valuation index for cardiorespiratory function in Students’ Physical Health Standard.” Sport Science. No. 11, pp.75-79. 2006.

[12] J. L. Ding. “An analysis of the existing problems of current reporting software for National Students’ Physical Health Standard.” Journal of Capital Institute of Physical Education and Sports. No. 1, pp.94-96, 2010.

[13] F. L. Rui. "Data submitting error analysis of the national students' Physical Health test in 2010.” Journal of Tianshui Normal Institution. No. 2, pp.91-93, 2011. 